\title{
Effects of transcutaneous electrical nerve stimulation on physical symptoms in advanced cancer patients receiving palliative care
}

Short title: Effects of transcutaneous electrical nerve stimulation in cancer patients

Jiro Nakano, PT, $\mathrm{PhD}^{1}$; Koji Ishii, MD, $\mathrm{PhD}^{2}$, Takuya Fukushima, PT, $\mathrm{PhD}^{3}$; Shun Ishii, $\mathrm{PT}^{4}$; Kazumi Ueno, Ns, $\mathrm{PhD}^{1}$; Emi Matsuura, Ns, $\mathrm{PhD}^{1}$; Kaori Hashizume, Ns, $\mathrm{PhD}^{1}$; Satoru Morishita, Ns, $\mathrm{MS}^{2}$; Koji Tanaka, OT, $\mathrm{PhD}^{5}$, Yoko Kusuba, Ns, $\mathrm{PhD}^{6}$

${ }^{1}$ Institute of Biomedical Sciences (Health Sciences), Nagasaki University (1-7-1

Sakamoto, Nagasaki-shi, Nagasaki 852-8520, Japan)

${ }^{2}$ Palliative Care Team, Nagasaki University Hospital (1-7-1 Sakamoto, Nagasakishi, Nagasaki 852-8501, Japan)

${ }^{3}$ Department of Rehabilitation Medicine, National Cancer Center Hospital (5-1-1 Tsukiji, Chuou-ku, Tokyo 104-0045, Japan)

${ }^{4}$ Department of Physical Therapy, Michino-Miyata Orthopedic Clinic (8-2 Koudagou, Nagayo-chou, Nishisonogi-gun, Nagasaki 851-2127, Japan)

${ }^{5}$ Graduate School of Health Sciences, Gunma University (39-22, 3 chome, Showa-machi, Maebashi, Gunma 371-8514, Japan)

${ }^{6}$ Department of Nursing Sciences, International University of Health and Welfare (1-7-1

Momochihama, Sawara-ku, Fukuoka 286-0048, Japan)

\section{Corresponding author:}

Jiro Nakano, PT, PhD

Institute of Biomedical Sciences (Health Sciences), Nagasaki University

Address: 1-7-1 Sakamoto, Nagasaki-shi, Nagasaki 852-8520, Japan.

Telephone and Fax: +81-95-819-7919

E-mail: nakano-j@nagasaki-u.ac.jp 


\begin{abstract}
Transcutaneous electrical nerve stimulation (TENS) is primarily used for pain, but might be useful for various other physical symptoms, including nausea, fatigue, dyspnea, and constipation. However, few studies have used TENS for treating the physical symptoms of patients with advanced cancer. In this crossover trial, we assess the effects of TENS on pain and other physical symptoms in 20 in-patients with advanced cancer receiving palliative care. For 5-day phases between wash out periods of 5 days, patients received TENS or non-TENS. TENS was delivered at four points: the center of the back for mainly nausea and dyspnea, on the back at the same dermatomal level as the origin of the pain $(100 \mathrm{~Hz})$, and on both ankle joints for constipation (10 $\mathrm{Hz}$ ). The intensity of pain and the total opioid dose used during phases were recorded. Physical symptoms were evaluated using the European Organization for Research and Treatment of Cancer (EORTC) Quality of Life Questionnaire Core 15 Palliative Care (QLQ-C15-PAL). Hematological and biochemical data were recorded before and after the TENS phase. The average pain and total number of opioid rescue doses were significantly reduced by TENS. TENS tended to improve nausea and appetite loss, but not constipation. There were no effects on hematological and biochemical parameters. Use of TENS could safely improve pain, nausea, and appetite loss in patients with advanced cancer. Although it cannot be used as a substitute for opioids and other pharmaceutical treatment, it may be useful to support palliative care.
\end{abstract}

Keywords: advanced cancer, transcutaneous electric nerve stimulation, pain, nausea, appetite loss, palliative care 


\section{Introduction}

Patients with advanced cancer usually have many symptoms that impair their quality of life. Pain can become unbearable and markedly reduces their quality of life (QOL) (Gordon, 1997). Opioids are a cornerstone of treatment for moderate to severe cancer pain (Caraceni et al., 2012). In most cases, cancer pain is persistent and requires chronic opioid treatment, and therefore management is extremely important. However, opioid side effects, such as nausea, vomiting, and constipation, may be the limiting factors in opioid use and could lead to early discontinuation and inadequate analgesic efficacy (Yamada $e t$ al., 2018). Therefore, to achieve good pain management in patients with cancer, it is necessary to minimize both pain and opioid side effects. Moreover, other physical symptoms, including fatigue, appetite loss, insomnia, and dyspnea, which are due to the tumor, chemotherapy (Yamagishi et al., 2009), and/or psychological distress (Chen and Chang, 2004), are frequent problems in advanced cancer patients. To manage these physical symptoms, pharmacotherapy and non-pharmacotherapy are provided as palliative care.

In terms of non-pharmacotherapy, the effects of massage (Wang et al., 2018), aromatherapy (Blackburn et al., 2017), relaxation and guided imagery (Baider et al., 1994), and music therapy (Gallagher et al., 2017) have all been reported, although the evidence for these treatments are not yet clear. Transcutaneous electrical nerve stimulation (TENS) can potentially also be used as an adjunct for the treatment of cancer pain. TENS is beneficial in the treatment of pain, including acute (Rakel et al., 2014) and chronic (Zeng et al., 2014) pain, in orthopedics. In terms of cancer pain, a few reports have shown the beneficial effect of TENS (Ahmed et al., 1998; Robb et al., 2007; Bennett et al., 2009; Loh and Gulati, 2013) against pain due to cancer in various organs. However, no conclusive evidence has been obtained as studies to date have used insufficient sample sizes (Loh and Gulati, 2014); thus, the accumulation of data is imperative.

The usefulness of TENS for cancer patients may involve not only pain reduction, but also alleviation of other physical symptoms during palliative care. For example, the effect of TENS on fatigue, nausea, and vomiting in cancer patients has been reported (Xie et al., 2017; Guo and Wang, 2018; Lee et al., 2019). The effect of TENS on other symptoms have also been reported, although not in cancer patients. Lau and Jones (Lau and Jones, 2008) reported that TENS improved dyspnea in patients with chronic obstructive pulmonary disease, while Iqbal et al. (2015) reported that TENS was effective in patients with chronic constipation. Yet, it has not been examined whether these effects could be obtained in advanced cancer patients. Additionally, it is possible that TENS can reduce further symptoms directly/indirectly, as different symptoms as well as emotions are 
interrelated in cancer patients (Zucca et al., 2012; McFarland et al., 2018).

This pilot study assessed the effects of TENS on pain and other physical symptoms, including pain, fatigue, vomiting/nausea, dyspnea, insomnia, appetite loss, and physical and emotional function in advanced cancer patients receiving palliative care.

\section{Methods}

\section{Study design}

This study employed a crossover design involving TENS and non-TENS phases in inpatients with advanced cancer who were undergoing palliative treatment at Nagasaki University Hospital. The Nagasaki University Hospital Ethics Committee approved this study (approval number: 17082103). Written informed consent was obtained from all participants before they participated in this study.

Any participants meeting the eligibility criteria for palliative care were recruited. After screening and enrolment, the participants were randomly allocated to groups A and B. Treatment sequence was assigned at random by using a randomized envelope method. The two-arm crossover trial consisted of a first period, wash-out period, and a second period. The TENS intervention (TENS phase) lasted 5 days and was administered during the first period in group A and during the second period in group B. Both groups received the usual care and palliative care during all periods in the study. The wash-out period was 5 days. Evaluations were performed before and after each phase.

\section{Participants}

Twenty-four patients were recruited from the Nagasaki University Hospital. Patients were eligible for inclusion if they had advanced cancer and cancer-related pain and were receiving palliative care; cancer affecting any organ was included. Cancer-related pain was defined as cancer-related nociceptive, neuropathic pain, but not orthopedic and dental pain. Patients were excluded if they refused participation in this study, were younger than 20 years, had a pacemaker, ischemic heart disease, electrical hypersensitivity, epilepsy, or skin lesions on the treatment sites (back and ankle), were considered to be unsuitable for participation in the study by the attending doctor, or were unable to communicate with the study evaluator.

\section{TENS application protocol}

TENS was applied to four sites by a medical researcher depending on the patient's pain and physical symptoms. The TENS device (Cefar Complex rehab 400, DJO France SAS, Mouguerre, France) had a 4-channel stimulator and there were 4 pairs of self- 
adhesive TENS stimulating electrodes (Dura-Stick plus, $5 \times 9 \mathrm{~cm}$, DJO Global, Vista, CA, USA). For placement of the gel pads, a neurotomal pattern was utilized (Loh and Gulati, 2013). For treatment of pain, one pair of gel pads were placed on the back at the dermatomal level that corresponded to the painful part or internal organ. Two pairs of gel pads were placed on the back at the $\mathrm{C} 7$ to Th8 dermatomal level, with a view to obtaining a relaxation effect that might relieve physical symptoms, such as nausea, vomiting, and dyspnea (Lau and Jones, 2008). All pairs of gel pads were placed so as to sandwich the spinal column. One pair of gel pads was placed behind the medial malleolus around the ankle joint, with a view to relieving constipation by stimulating the tibial nerve (Iqbal et al., 2015). When there was metastatic spinal tumor on the vertebra, the gels pads were placed longitudinally, to avoid stimulating the tumor directly. In TENS, high-frequency $(100 \mathrm{~Hz})$ stimulation was used for all treatments except for constipation (Loh and Gulati, 2013; Loh and Gulati, 2014). For constipation, low-frequency (10 Hz) was offered (Lau and Jones, 2008; Iqbal et al., 2015). Intensity was increased until the electrical sensation was strong but still comfortable. TENS was delivered for 30 minutes, once a day, by a physical therapist.

\section{Outcome measures}

Pain and physical symptoms were evaluated 1 day before and after the TENS and non-TENS phases. Researchers evaluated the patient at similar times of the day to ensure that the time from last dose of analgesia did not vary between the before and after period. Patients were given two pain questionnaires that incorporated a numerical rating pain scale (NRS): 1) the pain intensity at the actual time of evaluation, 2) the average pain intensity during the day. Additionally, on the first day of the TENS phase, the immediate effect of TENS was examined: current pain was evaluated by NRS immediately before TENS (baseline) and at $0 \mathrm{~min}, 60 \mathrm{~min}$, and $120 \mathrm{~min}$ after TENS administration.

Information about prescription opioid and evacuation were copied from the clinical record. The drug name and total daily dose of prescribed opioid (/day) were recorded and the dose was converted to morphine-equivalents (mg/day) (Treillet et al., 2018) at the time point of evaluation. For opioid rescue dose, the number of times that opioid needed to be taken for breakthrough pain was recorded during the intervention phase, regardless of the dose per time: the total number of times of using an opioid rescue dose in the TENS/non-TENS phase was examined. The total number of times of evacuation during the TENS/non-TENS phase was also counted to evaluate the effect on constipation.

To evaluate physical symptoms, the European Organization for Research and Treatment (EORT) of Cancer Quality of Life Questionnaire-Core 15-Palliative Care 
(QLQ-C15-PAL) was used (Groenvold et al., 2006). The QLQ-C15-PAL includes questions to evaluate pain, fatigue, nausea/vomiting, appetite loss, dyspnea, insomnia, constipation, and physical and emotional function. The scores of all symptoms and functions were calculated whereby higher symptom scale scores $(0-100)$ indicate worse status and higher functional scores (0-100) indicate better status. Patients filled out the question papers for themselves.

To confirm the safety of TENS for patients with cancer, blood data, including white and red blood cell counts, platelet count, lymphocyte count, and C-reactive protein, hemoglobin, and albumin levels, were recorded.

\section{Statistical analyses}

Data were expressed as mean and standard deviation (SD), or number and percentage of participants, as appropriate. The Wilcoxon signed-rank test was used to analyze preand post-intervention changes in pain, fatigue, nausea/vomiting, appetite loss, dyspnea, insomnia, and constipation, daily dose of prescribed opioid, total number of times of using opioid rescue dose, and number of evacuations. To examine the immediate effect of TENS on pain, we used one-way repeated-measures ANOVA for differences across time, followed by Sidak's multiple-comparison test. $P<0.05$ was considered statistically significant. Data were analyzed using the Statistics version 23 software (IBM SPSS, New York, USA).

\section{Results}

\section{Subjects}

Between October 2017 and January 2019, 24 patients with advanced cancer receiving palliative care were recruited. Four subjects withdrew during the study due to discharge $(\mathrm{n}=2)$, death $(\mathrm{n}=1)$, and deteriorating performance status $(\mathrm{n}=1)$. Finally, 20 patients completed the study. The baseline characteristics of these 20 patients are shown in Table 1.

\section{Effect of TENS on pain and opioid}

The immediate effect of TENS on pain is shown in Figure 1. One-way repeated measures ANOVA for time revealed a significant effect of time $\left(F_{1,57}=8.9 ; p<0.01\right)$. Pain immediately after TENS was significantly reduced compared with immediately before TENS (baseline) $(95 \% \mathrm{CI}=0.9,4.2 ; \mathrm{p}<0.01)$. However, this effect disappeared after 60 and $120 \min (\mathrm{p}=0.26 ; \mathrm{p}=0.99$, respectively).

The average and maximum pain NRS score during the day, the total dose of prescribed 
opioid per day, and the total number of rescue doses required during the TENS and nonTENS phases are shown in Table 2. When the average pain NRS score during the day were compared between pre- and post- intervention, there was a significant reduction in the score during the TENS-phase (95\%CI: $-3.14,-0.7 ; p<0.01)$, but not in the non-TENS phase. The change in average of pain during the TENS phase was significantly greater than during the non-TENS phase $(95 \% \mathrm{CI}:-4.0,-0.1 ; p=0.04)$. The carryover effect (95\%CI: $-4.9,1.4 ; p=0.25)$ and period effect (95\%CI: $-3.6,2.7 ; p=0.78)$ was not observed. In terms of the maximum pain during the day, there was no significant change between pre- and post-intervention in either the TENS or non-TENS phases. The daily dose of prescribed opioid was also not changed significantly in the TENS and non-TENS phases. However, the total number of times of requiring opioid rescue during the TENS phase (11.7 \pm 11.6 times) was reduced significantly as compared with that during the nonTENS phase (16.3 \pm 15.8 times) (Figure 2).

\section{Effect of TENS on physical symptoms and functions}

When the scores of physical symptoms were compared between pre- and postintervention, a significant reduction in pain (95\% $\mathrm{CI}$ : 14.0, 35.1; $p<0.01$ ), nausea/vomiting (95\%CI: 0.6, 24.3; $p=0.04)$, and appetite loss (95\%CI: 2.5, 20.8; $\mathrm{p}=$ 0.02 ) was shown in the TENS-phase, although symptoms were not changed in the nonTENS phase. For pain and nausea/vomiting, mean differences in the change value were confirmed. Functional scales in both physical and emotion functions were not changed in the TENS and non-TENS phases (Table 2).

The total number of evacuations in the TENS phase was not changed compared with that in the non-TENS phase (TENS: $6.8 \pm 4.9$ times; non-TENS: $6.9 \pm 4.9$ times; $p=$ 0.92 ). Because laxative was prescribed for four patients, the analysis was repeated after excluding their data, but the results were not statistically significant.

\section{Change of blood data}

There was no change in white and red blood cell counts, platelet count, lymphocyte count, or C-reactive protein, hemoglobin, albumin, or total protein levels between preand post-TENS phase. Additionally, no significant changes in other items of blood tests after TENS were found (data not shown).

\section{Discussion}

This randomized, crossover, pilot study assessed the effects of TENS on pain and other physical symptoms of advanced cancer patients undergoing palliative treatment. 
TENS significantly reduced the average pain and total number of times that opioid rescue treatment was required, and tended to improve nausea and appetite loss, without affecting hematological and biochemical parameters.

Generally, TENS is used for acute and chronic pain of muscles and joints (Rakel et al., 2014; Zeng et al., 2014). However, we sought to investigate the efficacy of TENS not only for pain, but also for other physical symptoms in advanced cancer patients, as there are some indications that TENS also has effects on fatigue, nausea/vomiting, constipation, and dyspnea (Cekmen et al., 2007, Lau and Jones, 2008; Iqbal et al., 2015; Lee et al., 2019). To this end, TENS was administered simultaneously to multiple areas of the body. This has not been reported previously, and could help to identify an optimal TENS method for multiple symptoms in advanced cancer patients.

We expected that TENS would at least reduce cancer pain. Although the mechanism underlying pain reduction by TENS has not been completely clarified, it is thought that TENS produces anti-hyperalgesia through opioid receptors, based on animal experiments (Sluka et al., 1999). Therefore, both nociceptive and neuropathic pain, including visceral pain, should be reduced, similar to the response to opioids. The opioid receptor that responds to TENS differed according to the frequency of electrical stimulation. The 100 $\mathrm{Hz}$ frequency used in this study might stimulate the $\delta$ opioid receptor (Sluka et al., 1999). Current pain was reduced by TENS immediately, but the effect did not persist for longer than 1 hour. The average pain during the day was also reduced significantly. Because the total daily dose of prescribed opioid was not changed, the reduction in the average pain during the day may be due to the effect of TENS.

These results support the findings of a previous study that showed the effect of TENS on cancer pain (Bennett et al., 2009; Loh and Gulati, 2013; Lee et al., 2019). Additionally, although the maximum pain during the day, which might reflect breakthrough pain due to the tumor, remained unchanged, the total number of times a rescue dose of opioid was required during the TENS phase ( 5 days) was decreased significantly. The pain symptom scale in the QLQ-C15-PAL also decreased significantly. We considered that these results showed that TENS treatment might lead to pain improvement partly based on psychological or emotional factors. The pain symptom is strongly correlated to psychological distress factors, such as anxiety and depression (Hassanein et al., 2005; Nicklasson and Bergman, 2007). However, it remains possible that TENS had a placebo effect, because we did not perform sham TENS during the non-TENS phase. Nevertheless, our results suggested that TENS was efficacious against pain in advanced cancer patients, even if it was due to a placebo effect. This supports the use of TENS as adjunctive treatment during palliative care in advanced cancer patients. 
Moreover, significant improvement in nausea/vomiting were shown in the TENS phase, as expected from previous studies (Xie et al., 2017; Guo and Wang, 2018). Additionally, TENS resulted in a significant improvement in regaining appetite between pre- and post-intervention, although there was no significant difference in change between the TENS and non-TENS phases. Improvement in appetite loss might be a secondary effect due to the reduction in nausea/vomiting, because there is a marked correlation between appetite loss and nausea/vomiting (Bener et al., 2018). Nausea/vomiting and appetite loss are important issues in advanced cancer patients receiving opioid and chemotherapy. In terms of the mechanism, it could be postulated that TENS affects nausea/vomiting via the same mechanism as opioid antiemetics and endogenous opioid peptides (Cekmen et al., 2007). Saller et al. further proposed that endogenous neuropeptides and serotoninergic mechanisms may be involved in the antiemetic effects of TENS because of their impact on gastrointestinal activity (Saller et al., 1986).

The expected improvements on physical symptoms, such as fatigue, dyspnea, and constipation, were not shown in this study. Only one previous study has reported a reduction of fatigue by TENS in head and neck cancer patients (Lee et al., 2019); this effect was not seen in the present study, for unclear reasons. This discrepancy may be due to differences in the frequency of electrical stimulating: this study adopted $100 \mathrm{~Hz}$, given the TENS machine used, while the previous study used TENS at $4 \mathrm{~Hz}$ (Lau and Jones, 2008); the influence of TENS on the central nervous system differs between low- and high-frequency stimulation (Sluka et al., 1999). In terms of constipation, several reports have shown the benefit of tibial or sacral nerve stimulation by TENS for chronic constipation (Iqbal et al., 2015; Ng et al., 2016; Yik et al., 2016). However, other reports have found no effect of TENS on chronic constipation (Kumar et al., 2016; $\mathrm{Ng}$ et al., 2016). In this study, the effect of TENS on constipation, a side effect of opioid treatment in advanced cancer patients, was investigated, but no effect was found. Because advanced cancer patients who had constipation were prescribed laxative during palliative care, the effect of TENS was not clear. However, even when the influence of laxative was excluded, no effect of TENS on constipation was observed. The period of TENS might be too short to note an effect on constipation, as it has been reported that a short-term period of TENS treatment was ineffective against chronic constipation (Iqbal et al., 2016).

This study had some limitations. First, the sample size was small. This study was conducted at a single hospital, which may affect the generalizability of the results. Second, the primary cancer and pain type varied among participants; However, the crossover design might reduce the resulting bias ( $\mathrm{Wu}$ and Anthony, 2000). Third, the analgesic 
benefits of TENS are also greatest during stimulation (Desantana et al., 2008) and have a limited duration (similar to pharmacologic approaches), which was also confirmed in this study. The effect of TENS differs with the frequency of stimulation and the location of the electrode pads (Sluka et al., 1999). Future studies should avoid these limitations.

In summary, our results showed the positive effect of TENS on not only pain, but also physical symptoms, such as nausea/vomiting and appetite loss, when applied simultaneously to multiple areas of the body in advanced cancer patients, and it was also performed safely. However, no benefits were found in terms of fatigue, dyspnea, and constipation, even though such effects were expected. There may be more effective TENS administration methods in terms of electrical frequency and area of stimulation. Although TENS may not be suitable as a substitute for opioids and other pharmaceutical treatment, it may be useful tool for supporting palliative care.

\section{Conflict of interest}

The authors declare no potential conflicts of interest with respect to the research, authorship, and/or publication of this article.

\section{Acknowledgements}

The authors thank the members of the Palliative care team of Nagasaki University Hospital for their support. This study was supported in part by Japan Society for the Promotion of Science KAKENHI, grant number 18K10711.

\section{References}

Ahmed HE, Craig WF, White PF, Huber P (1998). Percutaneous electrical nerve stimulation (PENS): a complementary therapy for the management of pain secondary to bony metastasis. Clin J Pain 14:320-323.

Baider L, Uziely B, De-Nour AK (1994). Progressive muscle relaxation and guided imagery in cancer patients. Gen Hosp Psychiatry 16:340-347.

Bener A, Alsulaiman R, Doodson L, El Ayoubi HR (2018). An assessment of reliability and validity of the European Organization for Research and Treatment of Cancer Quality of Life Questionnaire C30 among breast cancer patients in Qatar. J Family Med Prim Care 6:824-831.

Bennett MI, Johnson MI, Brown SR, Radford H, Brown JM, Searle RD (2009). Feasibility study of Transcutaneous Electrical Nerve Stimulation (TENS) for cancer bone pain. J Pain 11:351-359. 
Blackburn L, Achor S, Allen B, Bauchmire N, Dunnington D, Klisovic RB, et al. (2017). The Effect of Aromatherapy on Insomnia and Other Common Symptoms Among Patients With Acute Leukemia. Oncol Nurs Forum 44:E185-E193.

Caraceni A, Hanks G, Kaasa S, Bennett MI, Brunelli C, Cherny N, et al. (2012). Use of opioid analgesics in the treatment of cancer pain: evidence-based recommendations from the EAPC. Lancet Oncol 13:e58-68.

Cekmen N, Salman B, Keles Z, Aslan M, Akcabay M (2007). Transcutaneous electrical nerve stimulation in the prevention of postoperative nausea and vomiting after elective laparoscopic cholecystectomy. J Clin Anesth 19:49-52.

Chen ML, Chang HK (2004). Physical symptom profiles of depressed and nondepressed patients with cancer. Palliat Med 18:712-718.

Desantana JM, Santana-Filho VJ, Sluka KA (2008). Modulation between high- and lowfrequency transcutaneous electric nerve stimulation delays the development of analgesic tolerance in arthritic rats. Arch Phys Med Rehabil 89:754-760.

Gallagher LM, Lagman R, Rybicki L (2017). Outcomes of Music Therapy Interventions on Symptom Management in Palliative Medicine Patients. Am J Hosp Palliat Care 35:250-257.

Gordon C (1997). The effect of cancer pain on quality of life in different ethnic groups: a literature review. Nurse Pract Forum 8:5-13.

Groenvold M, Petersen MA, Aaronson NK, Arraras JI, Blazeby JM, Bottomley A, et al. (2006). The development of the EORTC QLQ-C15-PAL: a shortened questionnaire for cancer patients in palliative care. Eur J Cancer 42:55-64.

Guo WC, Wang F (2018). Effect of nerve electrical stimulation for treating chemotherapyinduced nausea and vomiting in patients with advanced gastric cancer: A randomized controlled trial. Medicine (Baltimore) 97:e13620.

Hassanein KA, Musgrove BT, Bradbury E (2005). Psychological outcome of patients following treatment of oral cancer and its relation with functional status and coping mechanisms. J Craniomaxillofac Surg 33:404-409.

Iqbal F, Collins B, Thomas GP, Askari A, Tan E, Nicholls RJ, et al. (2015). Bilateral transcutaneous tibial nerve stimulation for chronic constipation. Colorectal Dis 18:173-178.

Iqbal F, Thomas GP, Tan E, Askari A, Dastur JK, Nicholls J, et al. (2016). Transcutaneous Sacral Electrical Stimulation for Chronic Functional Constipation. Dis Colon Rectum 59:132-139.

Kumar L, Liwanag J, Athanasakos E, Raeburn A, Zarate-Lopez N, Emmanuel AV (2016). Effectiveness of percutaneous tibial nerve stimulation in managing refractory 
constipation. Colorectal Dis 19:45-49.

Lau KS, Jones AY (2008). A single session of Acu-TENS increases FEV1 and reduces dyspnoea in patients with chronic obstructive pulmonary disease: a randomised, placebo-controlled trial. Aust J Physiother 54:179-184.

Lee JE, Anderson CM, Perkhounkova Y, Sleeuwenhoek BM, Louison RR (2019). Transcutaneous Electrical Nerve Stimulation Reduces Resting Pain in Head and Neck Cancer Patients: A Randomized and Placebo-Controlled Double-Blind Pilot Study. Cancer Nurs 42:218-228.

Loh J, Gulati A (2013). The use of transcutaneous electrical nerve stimulation (TENS) in a major cancer center for the treatment of severe cancer-related pain and associated disability. Pain Med 16:1204-1210.

Loh J, Gulati A (2014). Transcutaneous electrical nerve stimulation for treatment of sarcoma cancer pain. Pain Manag 3:189-199.

McFarland DC, Shaffer KM, Tiersten A, Holland J (2018). Physical Symptom Burden and Its Association With Distress, Anxiety, and Depression in Breast Cancer. Psychosomatics 59:464-471.

Ng RT, Lee WS, Ang HL, Teo KM, Yik YI, Lai NM (2016). Transcutaneous electrical stimulation (TES) for treatment of constipation in children. Cochrane Database Syst Rev 11:CD010873.

Nicklasson M, Bergman B (2007). Validity, reliability and clinical relevance of EORTC QLQC30 and LC13 in patients with chest malignancies in a palliative setting. Qual Life Res 16:1019-1028.

Rakel BA, Zimmerman MB, Geasland K, Embree J, Clark CR, Noiseux NO, et al. (2014). Transcutaneous electrical nerve stimulation for the control of pain during rehabilitation after total knee arthroplasty: A randomized, blinded, placebocontrolled trial. Pain 155:2599-2611.

Robb KA, Newham DJ, Williams JE (2007). Transcutaneous electrical nerve stimulation vs. transcutaneous spinal electroanalgesia for chronic pain associated with breast cancer treatments. J Pain Symptom Manage 33:410-419.

Saller R, Hellenbrecht D, Buhring M, Hess H (1986). Enhancement of the antiemetic action of metoclopramide against cisplatin-induced emesis by transdermal electrical nerve stimulation. J Clin Pharmacol 26:115-119.

Sluka KA, Deacon M, Stibal A, Strissel S, Terpstra A (1999). Spinal blockade of opioid receptors prevents the analgesia produced by TENS in arthritic rats. $J$ Pharmacol Exp Ther 289:840-846.

Treillet E, Laurent S, Hadjiat Y (2018). Practical management of opioid rotation and 
equianalgesia. J Pain Res 11:2587-2601.

Wang K, Qi S, Lai H, Zhu X, Fu G (2018). Clinical massage therapy for patients with cancerrelated fatigue protocol of a systematic review. Medicine (Baltimore) 97:e13440.

Wu LT, Anthony JC (2000). The use of the case-crossover design in studying illicit drug use. Subst Use Misuse 35:1035-1050.

Xie J, Chen LH, Ning ZY, Zhang CY, Chen H, Chen Z, et al. (2017). Effect of transcutaneous electrical acupoint stimulation combined with palonosetron on chemotherapyinduced nausea and vomiting: a single-blind, randomized, controlled trial. Chin $J$ Cancer 36:6.

Yamada M, Matsumura C, Jimaru Y, Ueno R, Takahashi K, Yano Y (2018). Effect of Continuous Pharmacist Interventions on Pain Control and Side Effect Management in Outpatients with Cancer Receiving Opioid Treatments. Biol Pharm Bull 41:858863.

Yamagishi A, Morita T, Miyashita M, Kimura F (2009). Symptom prevalence and longitudinal follow-up in cancer outpatients receiving chemotherapy. J Pain Symptom Manage 37:823-830.

Yik YI, Stathopoulos L, Hutson JM, Southwell BR (2016). Home Transcutaneous Electrical Stimulation Therapy to Treat Children With Anorectal Retention: A Pilot Study. Neuromodulation 19:515-521.

Zeng C, Li H, Yang T, Deng ZH, Yang Y, Zhang Y, et al. (2014). Electrical stimulation for pain relief in knee osteoarthritis: systematic review and network meta-analysis. Osteoarthritis Cartilage 23:189-202.

Zucca AC, Boyes AW, Linden W, Girgis A (2012). All's well that ends well? Quality of life and physical symptom clusters in long-term cancer survivors across cancer types. $J$ Pain Symptom Manage 43:720-731. 
Table 1. Characteristics of participants.

BMI: body mass index; ECOG: Eastern Cooperative Oncology Group

Table 2. Comparison in the outcomes of pain and physical symptoms between preand post-intervention

The European Organization for Research and Treatment of Cancer Quality of Life Questionnaire-Core 15-Palliative Care (QLQ-C15-PAL): higher symptom scale scores $(0-100)$ indicate worse status and higher functional scores $(0-100)$ indicate better status.

Fig. 1 The immediate effect of TENS for pain (NRS)

TENS: transcutaneous electrical nerve stimulation. NRS: numerical rating pain scale. *: significant difference compared with Pre-TENS $(\mathrm{p}<0.05)$

Fig. 2 Comparison in total number of the rescue dose between pre- and postintervention

TENS: Transcutaneous electrical nerve stimulation. 
Table 1. Characteristics of participants.

\begin{tabular}{|c|c|}
\hline Characteristics & Value \\
\hline age, years & $70.0 \pm 6.3$ \\
\hline \multicolumn{2}{|l|}{ Sex, n $(\%)$} \\
\hline Male & $17(85.0)$ \\
\hline Female & $3(15.0)$ \\
\hline Hight $(\mathrm{cm})$ & $160.2 \pm 7.3$ \\
\hline Weight (Kg) & $53.9 \pm 5.7$ \\
\hline BMI & $20.9 \pm 3.14$ \\
\hline \multicolumn{2}{|l|}{ Primary cancer, n (\%) } \\
\hline Esophagus & $5(25.0)$ \\
\hline Head and Neck & $3(15.0)$ \\
\hline Breast & $3(15.0)$ \\
\hline Kidney & $2(10.0)$ \\
\hline Lymphoma & $2(10.0)$ \\
\hline Prostate & $1(5.0)$ \\
\hline Liver & $1(5.0)$ \\
\hline Lung & $1(5.0)$ \\
\hline
\end{tabular}


Stage of tumor, n (\%)

IV

$17(85.0)$

III

$3(15.0)$

Pain type, n (\%)

Nociceptive

Neuropathic

Disease duration, days

Days since hospitalization, days

Treatment, n (\%)

Chemotherapy

Radiotherapy

Chemo + Radiotherapy

ECOG Performance status, n (\%)
$17(85.0)$

$3(15.0)$

$477.0 \pm 534.8$

$27.7 \pm 34.0$

$6(30.0)$

$2(10.0)$

$12(60.0)$

$5(25.0)$

$12(60.0)$

Score 3

$3(15.0)$

Score 4

(15.0)

BMI: body mass index; ECOG: Eastern Cooperative Oncology Group 
Table 2. Comparison in the outcomes of pain and physical symptoms between pre- and post-intervention

\begin{tabular}{|c|c|c|c|c|c|c|c|c|}
\hline & \multicolumn{3}{|c|}{ TENS phase } & \multicolumn{3}{|c|}{ non-TENS phase } & \multirow{2}{*}{$\begin{array}{l}\text { Mean difference of } \\
\text { change }(95 \% \mathrm{CI})\end{array}$} & \multirow[b]{2}{*}{ p-value } \\
\hline & Pre & Post & Change & Pre & Post & Change & & \\
\hline Average pain $(0-10)$ & $5.9 \pm 2.2$ & $3.8 \pm 2.3$ & $-1.9 \pm 2.6^{*}$ & $5.3 \pm 2.3$ & $5.1 \pm 1.9$ & $0.2 \pm 2.2$ & $-2.1(-4.0,-0.1)$ & 0.04 \\
\hline Maximum pain $(0-10)$ & $6.9 \pm 2.2$ & $5.9 \pm 2.8$ & $-1.0 \pm 2.3$ & $5.8 \pm 2.8$ & $5.7 \pm 2.4$ & $-0.1 \pm 2.5$ & $-0.9(-2.7,0.9)$ & 0.31 \\
\hline Opioid (mg/day) & $25.1 \pm 17.9$ & $30.0 \pm 23.5$ & $6.9 \pm 15.4$ & $22.5 \pm 18.7$ & $31.6 \pm 19.4$ & $9.20 \pm 19.8$ & $-2.3(-14.0,9.3)$ & 0.69 \\
\hline \multicolumn{9}{|l|}{ QLQ-C15-PAL (0-100) } \\
\hline Pain & $58.4 \pm 23.9$ & $33.8 \pm 26.3$ & $-24.6 \pm 22.5 *$ & $54.6 \pm 29.8$ & $51.2 \pm 21.5$ & $-3.3 \pm 26.0$ & $-21.3(-40.1,-2.1)$ & 0.03 \\
\hline Fatigue & $38.8 \pm 20.3$ & $35.6 \pm 22.6$ & $-0.3 \pm 24.5$ & $34.9 \pm 22.1$ & $41.0 \pm 22.1$ & $6.1 \pm 28.5$ & $-6.4(-26.0,13.2)$ & 0.50 \\
\hline Nausea/vomiting & $28.3 \pm 35.1$ & $15.8 \pm 26.8$ & $-12.5 \pm 25.3 *$ & $21.7 \pm 27.6$ & $24.2 \pm 26.2$ & $2.5 \pm 19.0$ & $-15.0(-30.0,-0.1)$ & 0.05 \\
\hline Appetite loss & $45.0 \pm 31.1$ & $33.3 \pm 30.6$ & $-11.7 \pm 19.6 *$ & $45.0 \pm 33.3$ & $50.0 \pm 33.3$ & $4.9 \pm 34.7$ & $-16.7(-36.0,2.6)$ & 0.08 \\
\hline Dyspna & $25.0 \pm 21.3$ & $20.0 \pm 31.3$ & $-5.0 \pm 22.3$ & $25.0 \pm 30.3$ & $18.3 \pm 17.0$ & $-6.7 \pm 25.6$ & $1.7(-18.7,22.2)$ & 0.87 \\
\hline
\end{tabular}


Constipation

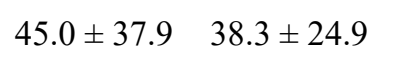

Physical function

$61.3 \pm 26.6 \quad 68.6 \pm 26.7$

$7.3 \pm 18.5$

$60.3 \pm 31.8$

$58.7 \pm 30.2$

$-1.7 \pm 14.7$

$9.0(-1.8,19.7)$

0.10

Emotion function

$71.7 \pm 26.4 \quad 68.8 \pm 30.6$

$-2.9 \pm 23.1$

$67.5 \pm 31.2 \quad 74.6 \pm 21.0 \quad 7.1 \pm 25.5$

$-10.0(-30.4,10.4)$

0.32

The European Organization for Research and Treatment of Cancer Quality of Life Questionnaire-Core 15-Palliative Care (QLQ-C15PAL): higher symptom scale scores $(0-100)$ indicate worse status and higher functional scores $(0-100)$ indicate better status. 


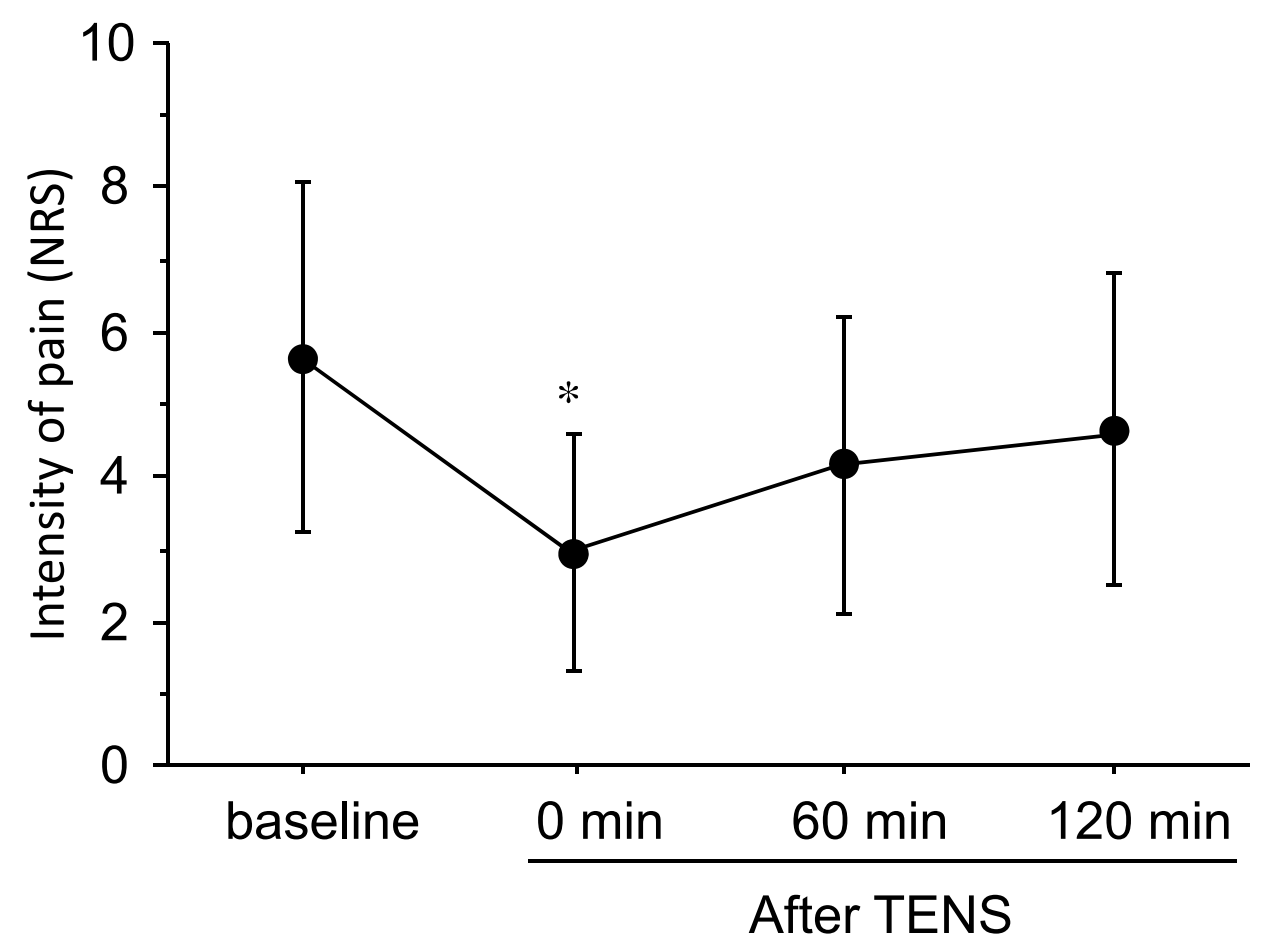

Fig. 1 The immediate effect of TENS for pain (NRS)

TENS: transcutaneous electrical nerve stimulation. NRS: numerical rating pain scale. *: significant difference compared with Pre-TENS $(p<0.05)$ 


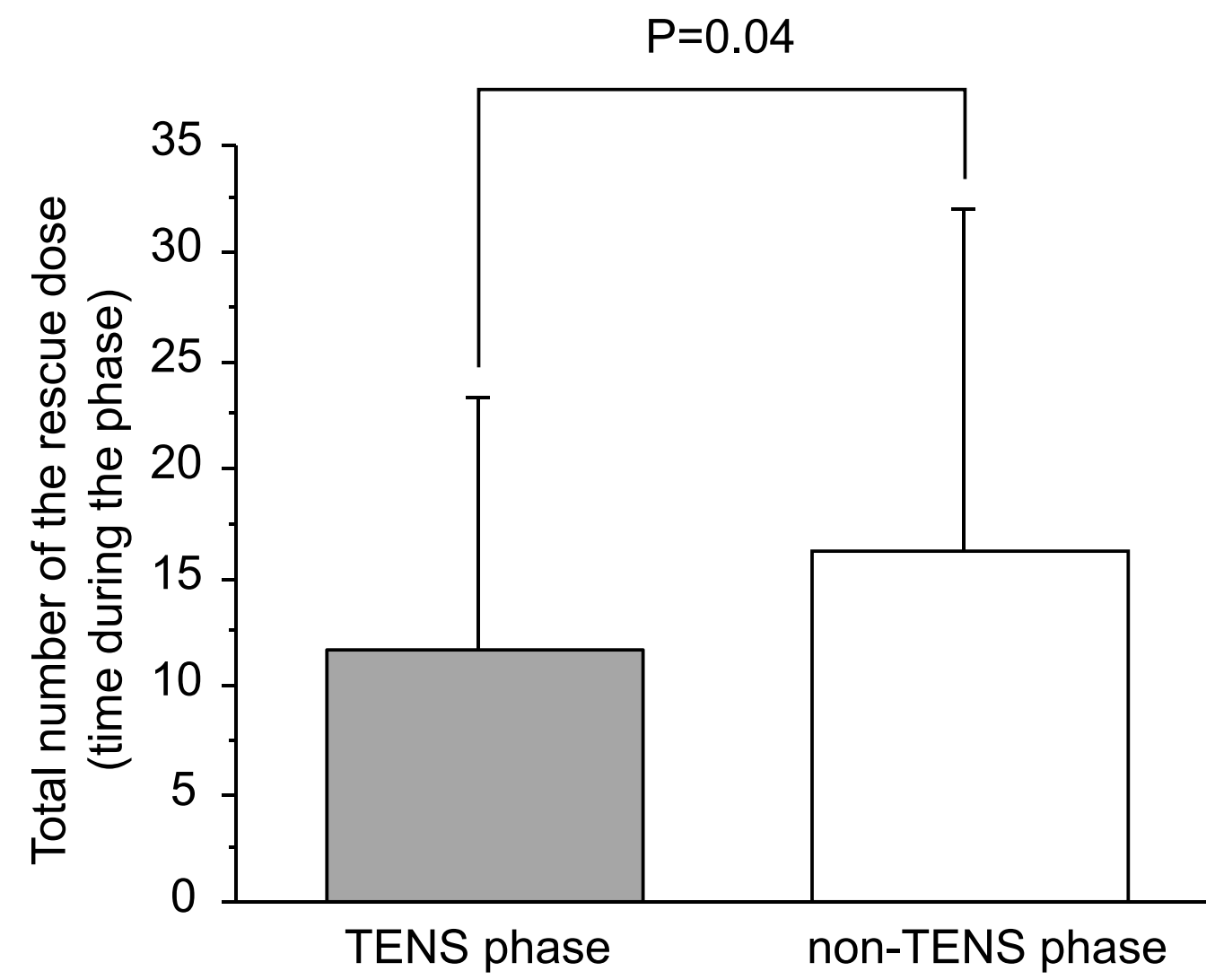

Fig. 2 Comparison in total number of the rescue dose between pre- and post-intervention

TENS: Transcutaneous electrical nerve stimulation. 